University of New Hampshire

University of New Hampshire Scholars' Repository

Physics Scholarship

Physics

10-15-2004

\title{
Mechanisms and dynamics of the metastable decay in Ar-2(+)
}

\author{
J. Fedor \\ K. Gluch \\ R. Parajuli \\ S. Matt-Leubner \\ Olof E. Echt \\ University of New Hampshire, Olof.Echt@unh.edu
}

See next page for additional authors

Follow this and additional works at: https://scholars.unh.edu/physics_facpub

Part of the Physics Commons

\section{Recommended Citation}

The following article appeared in J. Chem. Phys. 121, 7253 (2004); doi: 10.1063/1.1794011 and may be found at http://dx.doi.org/10.1063/1.1794011.

This Article is brought to you for free and open access by the Physics at University of New Hampshire Scholars' Repository. It has been accepted for inclusion in Physics Scholarship by an authorized administrator of University of New Hampshire Scholars' Repository. For more information, please contact Scholarly.Communication@unh.edu. 
Authors

J. Fedor, K. Gluch, R. Parajuli, S. Matt-Leubner, Olof E. Echt, P. Scheier, and T. D. Mark 


\section{AIP chemicical Physics}

Mechanisms and dynamics of the metastable decay in Ar2+

J. Fedor, K. Głuch, R. Parajuli, S. Matt-Leubner, O. Echt et al.

Citation: J. Chem. Phys. 121, 7253 (2004); doi: 10.1063/1.1794011

View online: http://dx.doi.org/10.1063/1.1794011

View Table of Contents: http://jcp.aip.org/resource/1/JCPSA6/v121/i15

Published by the American Institute of Physics.

Additional information on J. Chem. Phys.

Journal Homepage: http://jcp.aip.org/

Journal Information: http://jcp.aip.org/about/about_the_journal

Top downloads: http://jcp.aip.org/features/most_downloaded

Information for Authors: http://jcp.aip.org/authors
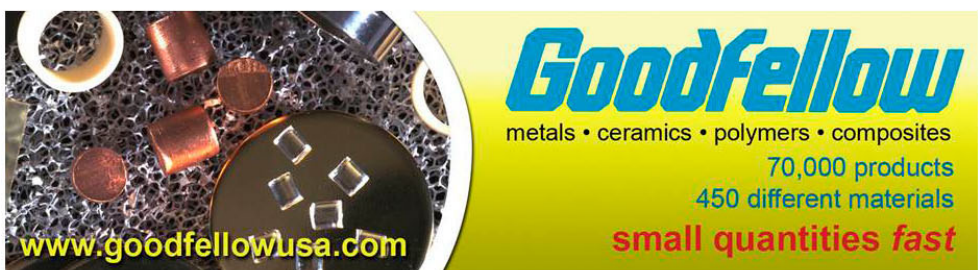


\title{
Mechanisms and dynamics of the metastable decay in $\mathrm{Ar}_{2}^{+}$
}

\author{
J. Fedor, K. Głuch, R. Parajuli, ${ }^{a}{ }^{a}$ S. Matt-Leubner, O. Echt, ${ }^{\text {b) }}$ P. Scheier, \\ and T. D. Märk ${ }^{\text {) }}$ \\ Institut für Ionenphysik, Leopold-Franzens Universität, Technikerstrasse 25, A-6020 Innsbruck, Austria
}

(Received 23 March 2004; accepted 26 July 2004)

\begin{abstract}
A detailed experimental as well as theoretical investigation of the properties of the metastable dissociation $\mathrm{Ar}_{2}^{+} \rightarrow \mathrm{Ar}^{+}+\mathrm{Ar}$ is presented. The mass-analyzed ion kinetic energy (MIKE) scan technique has been performed using a three sector field mass spectrometer. The possible mechanisms of the metastability of $\mathrm{Ar}_{2}^{+}$have been examined and the observed decay process is assigned to the $\mathrm{II}(1 / 2)_{u} \rightarrow \mathrm{I}(1 / 2)_{g}$ bound to continuum radiative transition, in agreement with earlier work. The calculation of the theoretical shape of the kinetic energy release distribution of fragment ions allowed us to construct the theoretical MIKE peak and compare it with the raw experimental data. The accuracy of various sets of potential energy curves for $\mathrm{Ar}_{2}^{+}$is discussed, as well as the way of production of the metastable $\operatorname{Ar}_{2}^{+}\left[\mathrm{II}(1 / 2)_{u}\right]$ electronic state by electron impact. Excellent agreement between the experimental data and theoretical model has been observed. (C) 2004 American Institute of Physics. [DOI: 10.1063/1.1794011]
\end{abstract}

\section{INTRODUCTION}

Molecular ions which have dissociative lifetimes in the time range $\geqslant 10^{-7} \mathrm{~s}$ are in mass spectrometry usually referred to as metastable ions. ${ }^{1}$ A very interesting and important feature of such ions is the decay mechanism, i.e., the reason for such delayed fragmentation. For larger systemspolyatomic molecular and cluster ions-the occurrence of vibrational (statistical) predissociation ${ }^{2}$ is very common; it may be treated in the framework of the quasiequilibrium theory. ${ }^{1,3}$ However, for smaller systems this mechanism is not operative; in the case of the diatomic ion the fragmentation would occur within one vibrational period-typically in the time domain of $10^{-15} \mathrm{~s}$. Other dissociation mechanisms have to be considered, and the potential energy curves for the particular system have to be consulted. Therefore the study of metastable decay of small ions, both from an experimental and theoretical point of view, is a very challenging domain of research.

Special attention has been paid to the dissociation of rare gas dimer ions. Rare gas dimer ions play an important role in excimer lasers where they are responsible for absorption losses; ${ }^{4,5}$ they occur also in high pressure lamps. ${ }^{6}$ The properties of rare gas dimer ions are also important for the modeling of larger cluster ions. ${ }^{7-10}$

The occurrence of the metastable dissociation $\mathrm{Ar}_{2}^{+}$ $\rightarrow \mathrm{Ar}^{+}+\mathrm{Ar}$ was first reported by Stephan et al. ${ }^{11}$ Using electron impact ionization of argon clusters and a double focusing two sector field mass spectrometer they were able to

\footnotetext{
${ }^{a)}$ Present address: Amrit Science Campus, Tribhuan University, Kathmandu, Nepal.

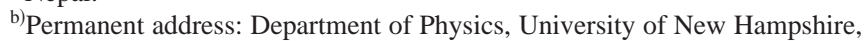
Durham, New Hampshire 03824.

c) Author to whom correspondence should be addressed; Adjunct professor at Department of Plasma Physics, Comenius University, SK-84248 Bratislava, Slovak Republic; Electronic mail: tilmann.maerk@uibk.ac.at
}

detect the metastable dissociation. In the pressure range of typically $5 \times 10^{-5}$ torr collision-induced dissociation (CID) $\mathrm{Ar}_{2}^{+}+\mathrm{Ar} \rightarrow \mathrm{Ar}^{+}+\mathrm{Ar}+\mathrm{Ar}$ was found to be the dominant source of $\mathrm{Ar}^{+}$, but from the dependence of the intensity ratio $i\left(\mathrm{Ar}^{+}\right) / i\left(\mathrm{Ar}_{2}^{+}\right)$on the background pressure and further extrapolation to zero pressure they concluded the occurrence of spontaneous metastable fragmentation. Stephan $e t$ al. also attempted a qualitative interpretation of the experimental results using the potential energy curves calculated by Wadt, ${ }^{12}$ suggesting the $\mathrm{I}(1 / 2)_{g}$ and $\mathrm{II}(1 / 2)_{u}$ electronic states to be unstable with respect to dissociation in the metastable time regime. However, in a later study by Whitaker et al. ${ }^{13}$ it was demonstrated that the suggested processes are either too slow or too fast to be detected in the mass spectrometer (see also the present calculations given below). In their follow-up study Stephan and Märk ${ }^{14}$ investigated decay reactions of $\mathrm{Ar}_{2}^{+}$produced by the autoionization sequence

$$
\begin{aligned}
& \mathrm{Ar}+e \rightarrow \mathrm{Ar}^{*}+e, \\
& \mathrm{Ar}^{*}+\mathrm{Ar} \rightarrow \mathrm{Ar}_{2}^{+}+e,
\end{aligned}
$$

finding a metastable fraction $i\left(\mathrm{Ar}^{+}\right) / i\left(\mathrm{Ar}_{2}^{+}\right)$being about a factor 100 larger than that for $\mathrm{Ar}_{2}^{+}$produced directly by electron impact ionization of argon clusters.

The photoion-photoelectron coincidence (PIPECO) study of Norwood et al. ${ }^{15}$ further elucidated the properties of this decay reaction. Their observation of the $\operatorname{Ar}_{2}^{+}\left[\mathrm{II}(1 / 2)_{u}\right]$ PIPECO electronic band was consistent with the interpretation that the $\operatorname{Ar}_{2}^{+}\left[\mathrm{II}(1 / 2)_{u}\right]$ is metastable with a radiative lifetime longer than their flight time of $\mathrm{Ar}_{2}^{+}(47 \mu \mathrm{s})$. They considered two dipole allowed transitions, II $(1 / 2)_{u}$ $\rightarrow \mathrm{I}(1 / 2)_{g}$ and $\mathrm{II}(1 / 2)_{u} \rightarrow \mathrm{I}(3 / 2)_{g}$. The calculated dissociative lifetimes were $90.9 \mu \mathrm{s}$ and $50.7 \mathrm{~s}$, respectively; thus Norwood et al. assigned the observed process to the first transition.

The most extensive analysis of the argon dimer ion dissociation has been presented by Whitaker et al. ${ }^{13}$ In addition 


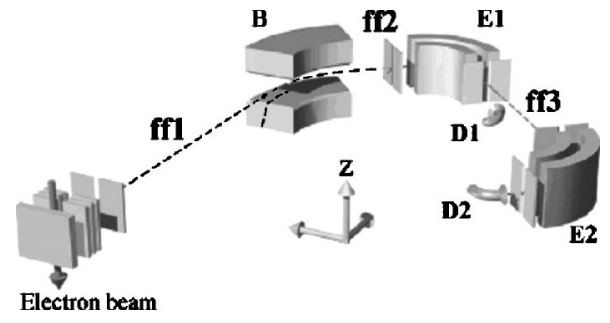

FIG. 1. Schematic drawing of the experimental setup.

to the experimentally determined distribution of the kinetic energies of fragment ions, Whitaker and co-workers showed that the $\mathrm{II}(1 / 2)_{u}$ electronic state is strongly populated in the ionization, and they assigned the observed $\mathrm{Ar}_{2}^{+}$decay to the $\mathrm{II}(1 / 2)_{u} \rightarrow \mathrm{I}(1 / 2)_{g}$ bound to continuum transition. In a follow-up paper Stace and co-workers ${ }^{16}$ investigated how $A r_{2}^{+}$in the $\mathrm{II}(1 / 2)_{u}$ electronic state is produced in the electron impact ion source. They arrived at the conclusion that up to a stagnation pressure in the cluster source of about 1.7 bar under their experimental conditions $(200 \mu \mathrm{m}$ conical nozzle, $300 \mathrm{~K}$ temperature of the neutral gas) the dominant means of the $\operatorname{Ar}_{2}^{+}\left[\mathrm{II}(1 / 2)_{u}\right]$ production is the vertical ionization of the neutral dimer; at higher pressure expulsion of the metastable dimer ion from larger cluster ions is taking over.

Yoshii and co-workers ${ }^{17}$ were the first to report metastable decay in other rare gas dimer ions, $\mathrm{Kr}_{2}^{+}$and $\mathrm{Xe}_{2}^{+}$. However, surprisingly they did not detect any decay of $\mathrm{Ar}_{2}^{+}$, contrary to other work mentioned above. A possible explanation for this disagreement will be presented below.

In a previous paper ${ }^{18}$ we reported on the metastable decay of $\mathrm{Ar}_{2}^{+}$and $\mathrm{Ne}_{2}^{+}$, finding strikingly different kinetic energy release distributions of the fragment ions. Furthermore, a theoretical approach explained the experimental data, and this comparison of theory with experiment turned out to be a sensitive test for the accuracy of the $a b$ initio potential energy curves. In this report we briefly review experimental results about the metastable dissociation of $\mathrm{Ar}_{2}^{+}$obtained using a double-focusing three sector field mass spectrometer in combination with the mass-analyzed ion kinetic energy (MIKE) scan technique and provide details about the theoretical treatment. We carefully examine possible mechanisms of the metastability of $\mathrm{Ar}_{2}^{+}$. For the only operative process, the II $(1 / 2)_{u} \rightarrow \mathrm{I}(1 / 2)_{g}$ bound to continuum transition, we calculate the kinetic energy release distribution (KERD); this allows comparison with the raw experimental data. Possible complications in the theoretical model are discussed and the effects of the internal temperature of clusters in the supersonic beam and the shape of the potential energy curves on the resulting KERD are described.

\section{EXPERIMENTAL SETUP}

Details about the experimental setup and analysis have been published elsewhere. ${ }^{19,20}$ Figure 1 shows a schematic view of the experimental setup. The beam of neutral clusters is produced by a supersonic expansion of argon gas through a $20 \mu \mathrm{m}$ nozzle. Stagnation pressure was $1 \mathrm{bar}$, temperature $-150^{\circ} \mathrm{C}$. The produced argon clusters are ionized by electron impact, the energy of the ionizing electrons being typi- cally $100 \mathrm{eV}$. Ions are then extracted and accelerated by $3 \mathrm{kV}$ before entering the mass spectrometer. They pass through the first field free region ( $\mathrm{ff} 1$, length $61 \mathrm{~cm}$ ), are momentum analyzed by a magnetic sector field $(B)$, enter a second fieldfree region (ff2, length $33.3 \mathrm{~cm}$ ), pass through a $90^{\circ}$ electric sector field $(E 1)$, enter a third field free region (ff3, length $92 \mathrm{~cm})$, pass through another electrostatic sector field ( $E 2$, geometry identical with $E 1$ ), and finally are detected by a channeltron-type electron multiplier D2. Alternatively, another channeltron $D 1$ may be moved into the ion beam to register all ions that pass through the exit slit of $E 1 . \mathrm{Ar}_{2}^{+}$ parent ions traverse ff 2 during the time interval $15.0 \leqslant t$ $\leqslant 19.2 \mu \mathrm{s}$ and ff3 during $25.0 \leqslant t \leqslant 30.3 \mu \mathrm{s}$.

The MIKE technique has been applied to investigate the delayed dissociation of argon dimer ions. In one of the two possible modes, we examine ion decay in ff2: The magnetic field is tuned so that only ions with the mass to charge ratio of the parent ion $\left(m_{p} / q_{p}\right)$ pass the $B$ sector, the $E 1$ sector field voltage is scanned, and the signal in $D 1$ is recorded as a function of this voltage. In the second operation mode, decay of $\mathrm{Ar}_{2}^{+}$is analyzed in ff3 by tuning the magnet to transmit the parent ion, setting the voltage of $E 1$ such that it transmits stable parent ions, and scanning the sector field voltage of $E 2$. The signal is recorded in $D 2$. In this mode, $B$ and $E 1$ constitute a double focusing high resolution mass spectrometer. In both modes, if $\mathrm{Ar}_{2}^{+}$decays in the field free region and $U_{p}$ denotes the voltage required to transmit a stable parent ion (typically $U_{p}=511 \mathrm{~V}$ in our system), then the electric sector will transmit fragment ions $\mathrm{Ar}^{+}\left(m_{f} / q_{f}\right)$ if the sector field voltage is set to

$$
U_{f}=\frac{m_{f} / q_{f}}{m_{p} / q_{p}} U_{p}
$$

i.e., $U_{f}=U_{p} / 2$ in the present case. Furthermore, due to the additional kinetic energy release, the fragment ion MIKE peak is always broadened. Simple kinematic considerations show ${ }^{1}$ that if all fragments had the same additional kinetic energy release (KER), the MIKE peak would have a rectangular shape with width $\Delta U_{f}$, where

$$
\mathrm{KER}=\frac{q_{f}^{2} m_{p}^{2} V_{a c c}}{16 q_{p} m_{f}^{2}}\left(\frac{\Delta U_{f}}{U_{f}}\right)^{2}=c \Delta U_{f}^{2} .
$$

If the fragments have a certain kinetic energy release distribution $n$ (KER), this distribution will be directly proportional to the first derivative of the fragment ion signal $I_{f}$ with respect to the sector field voltage

$$
n(\mathrm{KER})=-\operatorname{const} \frac{d I_{f}}{d U} .
$$

In the present work we calculate the theoretical $n$ (KER) and construct the fragment ion peak to compare with the experimental one. Relations (3) and (5) are valid only if the parent ion MIKE peak is infinitely narrow, i.e., for a $\delta$-function-like parent peak. In reality there is always a certain distribution of the parent ion signal and to obtain the fragment ion peak from the known $n$ (KER) we convolute the calculated fragment ion peak with the finite parent ion peak shape. 

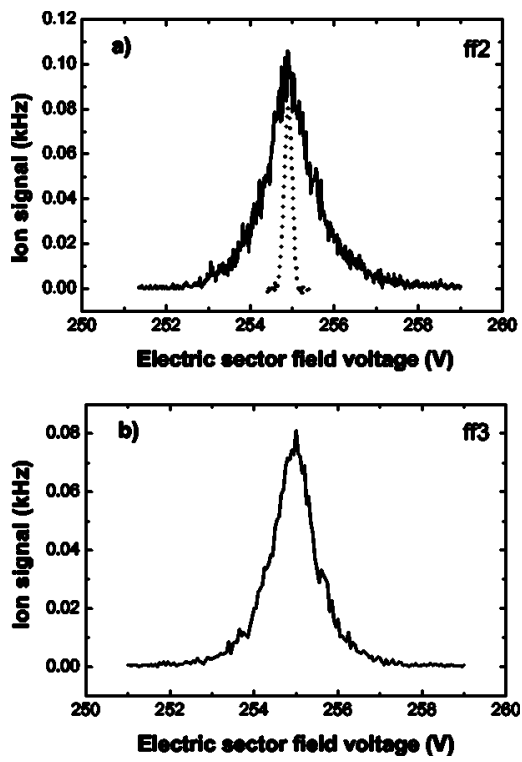

FIG. 2. Experimental fragment ion MIKE spectra recorded for the metastable decay reaction $\mathrm{Ar}_{2}^{+} \rightarrow \mathrm{Ar}^{+}+\mathrm{Ar}$ in ff2 (a) and ff3 (b). The dotted line in (a) is the parent ion peak scaled to the position of the fragment peak; its width reflects the experimental resolution.

\section{RESULTS}

\section{A. Experimental data and possible explanations}

Figure 2 shows the fragment ion MIKE spectra recorded in the two different experimental time windows. One can see that both peaks have virtually the same shape. This confirms the reliability of the experimental method and suggests that the kinetic energy release distribution does not change much within the time difference between the two field free regions. Additionally, panel (a) shows the expected shape of the fragment ion peak if the kinetic energy release were identical to zero; this is just the parent ion peak scaled to the voltage of the fragment ion peak (dotted line). The metastable fraction $\left[i\left(\mathrm{Ar}^{+}\right) / i\left(\mathrm{Ar}_{2}^{+}\right)\right]$is $\approx 2 \times 10^{-4}$ in both time windows.

First we check if there are contributions from CID to the recorded signal. Typical background pressure in the field free regions was $5 \times 10^{-8}$ mbar. Increase of the pressure by up to one order of magnitude resulted in no noticeable change in the shape of the MIKE peak and no change in the metastable fraction; this eliminates the occurrence of CID in the present experiment.

For the detailed analysis of the MIKE spectra, knowledge of the potential energy curves for the lowest electronic states of $\mathrm{Ar}_{2}^{+}$is required. There exists a wide interest in $a b$ inito calculations of these potential energy curves. Since the pioneering POL CI (polarization-configuration interaction) study by Wadt, ${ }^{12}$ larger and larger $a b$ initio treatments have been reported. Especially worth being mentioned are the density functional study by Michels and co-workers,${ }^{21}$ single reference CI work by Whitaker et al. ${ }^{16}$ and the MRCI treatment by Gadea and Paidarova. ${ }^{22}$ However, the most accurate $a b$ initio potential curves up to date are those by Ha et al. ${ }^{23}$ which were subsequently modified to fit the extensive experimental data set from the pulsed-field-ionization zerokinetic-energy photoelectron spectroscopy by Wüest and

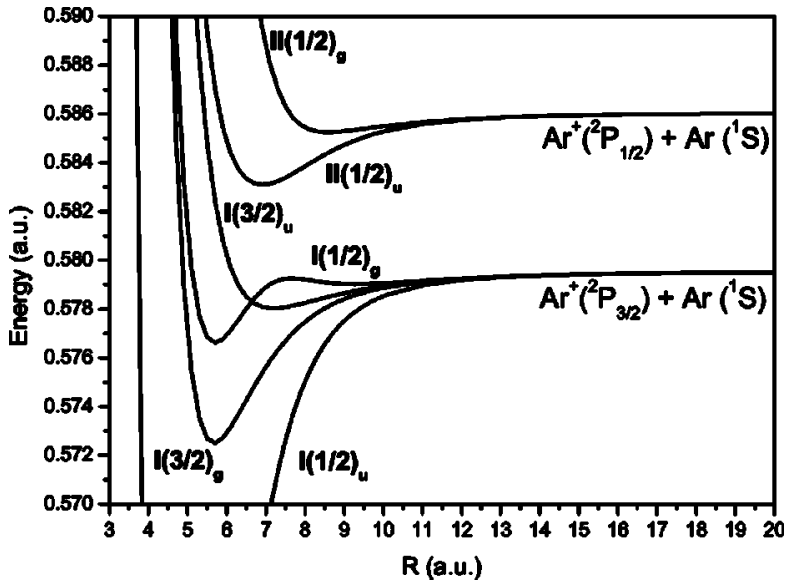

FIG. 3. Potential energy curves for the six lowest electronic states of $\mathrm{Ar}_{2}^{+}$ taken from Ref. 24.

Merkt. ${ }^{24}$ These curves are displayed in Fig. 3. They will be used for our analysis throughout this work unless stated otherwise. There is a striking difference between these curves and all previous ones that is of importance to our work. Namely, the local maximum in the $\mathrm{I}(1 / 2)_{g}$ potential curve is below the dissociation limit. This fact led Yoshii et al. ${ }^{17}$ to conclude that $\mathrm{Ar}_{2}^{+}$cannot dissociate via radiative transition to the continuum of this electronic state. As shown below, this statement is invalid.

It is worthwhile to examine in detail all possible processes which may lead to the delayed dissociation of the diatomic ion. The first possible process is tunneling through a barrier in the effective potential energy curve. For example, when the parent ion is in a high rotational state, the quasibound levels, or orbiting resonances, can dissociate in the time range of microseconds (rotational predissociation). This type of predissociation has been, for example, detected in the case of $\mathrm{HeH}^{+} .{ }^{25}$ The most likely candidate for this process in the case of $\mathrm{Ar}_{2}^{+}$is the $\mathrm{I}(1 / 2)_{g}$ electronic state, since it possesses a barrier even in the case of no rotation (due to the spin-orbit mixing of the bound ${ }^{2} \Pi_{g}$ and repulsive ${ }^{2} \Sigma_{g}$ states). To examine the possible occurrence of tunneling in this electronic state, we located the positions of the quasibound levels using the fact that the phase of the continuum nuclear wave function changes rapidly at the energy of the resonance ${ }^{26,27}$ Furthermore, we calculated lifetimes of these quasibound states using the semiclassical WKB approximation. ${ }^{28,26}$ The first quasibound states appear at relatively high rotational quantum numbers $(J=55)$, and their lifetimes are very short—up to $J=70$ they are $<10^{-8}$ s. Thus, within the accessible rotational states of $\operatorname{Ar}_{2}^{+}\left[\mathrm{II}(1 / 2)_{u}\right]$ (see below), tunneling happens too fast to be detected in our experiment.

The next possible $\mathrm{Ar}_{2}^{+}$metastable decay process might be electronic predissociation of the $\mathrm{II}(1 / 2)_{u}$ to the $\mathrm{I}(3 / 2)_{u}$ state. Such a nonradiative transition in the absence of curve crossing has been observed in $\mathrm{HeNe}^{+29}$ as well as in $\mathrm{Ne}_{2}^{+} \cdot{ }^{18}$ However, this process can be ruled out if we estimate the corresponding kinetic energy release. In the described predissociation the whole difference between the energy of the bound $\mathrm{II}(1 / 2)_{u}$ level and the asymptotic energy of the $\mathrm{I}(3 / 2)_{u}$ 
state would be released in the form of kinetic energy. This corresponds to roughly $100 \mathrm{meV}$. By taking the derivative of the measured MIKE peak (Fig. 2), properly deconvoluted with the parent ion peak, one obtains the experimental KERD (Ref. 18) with an average value of $19 \mathrm{meV}$. The large difference between expected and observed KER eliminates electronic predissociation as a viable decay process.

As suggested in previous work, ${ }^{13,15,18}$ the most probable reason for the metastability of $\mathrm{Ar}_{2}^{+}$is the $\mathrm{II}(1 / 2)_{u} \rightarrow \mathrm{I}(1 / 2)_{g}$ radiative transition. In this case, the final state lies in the continuum of the $\mathrm{I}(1 / 2)_{g}$ electronic state (above its dissociation limit); the difference between the energy of the initial bound rovibrational level of $\mathrm{II}(1 / 2)_{u}$ and the final state is radiated, and the energy difference between the final state and the $\mathrm{I}(1 / 2)_{g}$ asymptote is released in the form of kinetic energy of the fragments.

\section{B. II(1/2) $)_{u} \rightarrow I(1 / 2)_{g}$ bound to continuum transition}

The II $(1 / 2)_{u}$ electronic state is metastable-if it is populated in the ion source, all possible radiative transitions have low rates. Transitions into lower states with the same parity are forbidden, therefore very slow. The theoretical lifetimes for radiative transitions from the rovibrational states of $\mathrm{II}(1 / 2)_{u}$ into $\mathrm{I}(3 / 2)_{g}$ are of the order of seconds, ${ }^{13,15}$ this is confirmed also by our present calculations. The lifetime for the $\mathrm{II}(1 / 2)_{u} \rightarrow \mathrm{I}(1 / 2)_{g}$ transition is $2.5 \mu \mathrm{s}$ according to Whitaker (average value for a thermal population of rovibronic levels) and $50 \mu$ s according to Norwood ${ }^{15}$ (using the potential energy curves from Wadt, ${ }^{12}$ vibrational levels not specified). For the more recent set of potential energy curves $^{24}$ one can expect this continuum transition to be slower due to the lower local maximum in the $\mathrm{I}(1 / 2)_{g}$ electronic state. Evaluation of the transition rates using the potential energy curves from Wüest and Merkt ${ }^{24}$ together with the dipole transition moments from Gadea and Paidarova ${ }^{22}$ yield lifetimes of the rovibrational levels in the range of 100 to $300 \mu$ s except for the two lowest vibrational states, which have very poor overlap with the continuum wave functions and thus much longer lifetimes of $9.5 \mathrm{~ms}(v=0)$ and $2.5 \mathrm{~ms}(v=1)$. However, since there is no competing process-the calculated rates for transitions into bound states of $\mathrm{I}(1 / 2)_{g}$ are in all cases at least an order of magnitude lower-we can observe these continuum transitions in the mass spectrometer. And since the lifetimes are such that the relative population of the $\mathrm{II}(1 / 2)_{u}$ vibrational states does not change much between the different experimental time windows, there is no significant change in KERD expected, in agreement with the lack of change in the MIKE peaks in Fig. 2.

Hence, from a calculation of rates for transitions from the $\mathrm{II}(1 / 2)_{u}$ state we find that transitions into the continuum of $\mathrm{I}(1 / 2)_{g}$ can explain the observed metastable $\mathrm{Ar}_{2}^{+}$dissociation. Additionally, the MIKE scan technique offers a more sensitive test. If we calculate the KERD for the proposed decay process, we can construct the theoretical MIKE peak based on Eq. (5) and thus directly compare our experimental results with the theoretical predictions. The KERD for the decay from a single rovibrational level is simply given as the dependence of the Einstein coefficient for spontaneous emis- sion on the energy of the final state. Generally, the radiative transition rate is given by Fermi's golden rule: ${ }^{30}$

$$
A_{(v, J) \rightarrow\left(E, J^{\prime}\right)}=\frac{4}{3} \alpha \frac{\omega_{0}^{3}}{c^{2}}\left|M_{(v, J) \rightarrow\left(E, J^{\prime}\right)}\right|^{2} \rho(E) .
$$

Here $\omega_{0}$ is the energy of the emitted photon, $\alpha$ the finestructure constant, $M_{(v, J) \rightarrow\left(E, J^{\prime}\right)}$ the matrix element for the interaction expressing the coupling between the initial and the final state, and $\rho(E)$ is the density of final states at the energy of the final state. For radiative transitions in diatomic molecules the interaction operator is the electric dipole moment operator, and the matrix element after integrating out the electronic coordinates is

$$
M_{(v, J) \rightarrow\left(E, J^{\prime}\right)}=\delta_{J, J^{\prime}} \int_{0}^{\infty} \psi_{\left(E, J^{\prime}\right)}^{*}(R) D_{e}(R) \psi_{(v, J)}(R) d R,
$$

where $\psi_{(v, J)}$ is the nuclear wave function of the initial bound rovibrational state, $\psi_{\left(E, J^{\prime}\right)}$ is the continuum nuclear wave function of the final state and $D_{e}(R)$ is the electronic dipole matrix element for the $\mathrm{II}(1 / 2)_{u} \rightarrow \mathrm{I}(1 / 2)_{g}$ transition as a function of the internuclear distance that we take from Ref. 22. In the calculation we use the Q-branch approximation, this is expressed by $\delta_{J, J^{\prime}}$. The normalization of the continuum wave functions per unit energy and the corresponding expression for the density of states described in Ref. 31 lead to the final expression for the transition rate

$$
\begin{aligned}
A_{(v, J) \rightarrow(E, J)} & \\
= & \frac{4}{3} \frac{\omega_{0}^{3}}{c^{2}} \alpha \frac{\sqrt{2}}{\pi \hbar} \\
& \times \sqrt{\frac{\mu}{\operatorname{KER}}}\left(\int_{0}^{+\infty} \psi_{(E, J)}^{*}(R) D_{e}(R) \psi_{(v, J)}(R) d R\right)^{2} .
\end{aligned}
$$

Here $\mu$ is the reduced mass of the nuclei and KER is the kinetic energy of fragments corresponding to the final state. If we define the total decay rate of the particular rovibrational level $A_{(v, J)}=\int_{E_{\min }}^{E_{\max }} A_{(v, J) \rightarrow(E, J)} d E$, the intensity of the transition from the particular rovibrational level to the particular continuum energy at the time $t_{\exp }$ is given by

$$
\mathrm{I}\left(t_{\exp }\right)=N_{(v, J)}(0) A_{(v, J) \rightarrow(E, J)} e^{-A_{(v, J)} t_{\exp } .}
$$

Here $N_{(v, J)}(0)$ stands for the population of the $(v, J)$ level at the time of the ion production. Equation (9) expresses the KERD if only one particular rovibrational level of II $(1 / 2)_{u}$ is populated.

\section{Production of $\mathrm{Ar}_{2}^{+}$in the ion source}

To calculate the overall KERD one has to know the relative population of the rovibrational levels $N_{(v, J)}(0)$ in $\mathrm{II}(1 / 2)_{u}$. Here the mechanism of production of metastable $\mathrm{Ar}_{2}^{+}$in the ion source plays a role. One possibility is the direct ionization of the neutral dimer $\mathrm{Ar}_{2}+e \rightarrow \mathrm{Ar}_{2}^{+}$. However, Buck and Meyer ${ }^{32}$ showed in their scattering study that most of the $\mathrm{Ar}_{2}^{+}$in a typical supersonic beam $(200 \mu \mathrm{m}$ nozzle, 1.5 bar stagnation pressure) is produced by dissociative ionization of larger argon clusters leading to $\mathrm{Ar}_{n}+e$ 
$\rightarrow \mathrm{Ar}_{n}^{+} \rightarrow \mathrm{Ar}_{2}^{+}+(n-2)$ Ar. Such fragmentation of larger cluster ions happens immediately in the ion source. However, this experimental observation refers to the overall $\mathrm{Ar}_{2}^{+}$ signal. As to the content of $\mathrm{Ar}_{2}^{+}$in the metastable $\mathrm{II}(1 / 2)_{u}$ state, Stace et al. ${ }^{16}$ measured the metastable fraction $i\left(\mathrm{Ar}^{+}\right) / i\left(\mathrm{Ar}_{2}^{+}\right)$as a function of the argon stagnation pressure in the cluster source. They realized that starting from a certain pressure the metastable fraction increased with increasing pressure. They concluded that $\mathrm{Ar}_{2}^{+}\left[\mathrm{II}(1 / 2)_{u}\right]$ is produced via fragmentation of larger cluster ions only at sufficiently high argon stagnation pressure whereas at lower pressures it is produced directly by ionization of the argon dimer.

What mechanism dominates in our experiment can be examined using the scaling laws for production of clusters in supersonic expansion. Hagena ${ }^{33}$ showed that to have the same cluster size distribution in different supersonic experiments, the condition

$$
P_{0} D_{\text {eff }}^{1.5} T_{0}^{-2.4}=\text { const }
$$

has to be fulfilled. Here $P_{0}$ is the stagnation pressure in front of the nozzle, $D_{\text {eff }}$ is the effective nozzle diameter containing the nozzle shape factor, and $T_{0}$ is the temperature of the stagnant gas. Comparing our parameters with Stace's ${ }^{16}$ experiment one finds that in order to have the same cluster size distributions, the stagnation pressure in our experiment has to be a factor 15 higher than in the experiment of Stace et al. To have a significant contribution to metastable $\mathrm{Ar}_{2}^{+}$from fragmentation of larger cluster ions, our stagnation pressure would have to be around 25.5 bar, i.e., $\approx 25$ times the pressure actually used. Thus we conclude that ionization of the neutral argon dimer is the main source of metastable $\mathrm{Ar}_{2}^{+}$.

This conclusion may appear to be in disagreement with the strong fragmentation reported by Buck and Meyer. ${ }^{32}$ However, their results might apply only to $\mathrm{Ar}_{2}^{+}$in its ground electronic state. In this case, after vertical ionization of $\mathrm{Ar}_{n}$, a dimer ion core is formed in $\mathrm{Ar}_{n}^{+}$and the energy corresponding to the binding energy of $\mathrm{Ar}_{2}^{+}$is given to the rest of the cluster in the form of vibrational energy. ${ }^{34}$ For the ground electronic state of $\mathrm{Ar}_{2}^{+}$this is $1.36 \mathrm{eV}$ (Ref. 35) which leads to the immediate breaking of the bonds in the rest of the cluster ion. For II(1/2) $)_{u}$ the binding energy is only $50 \mathrm{meV}$ and the released energy after formation of such dimer ion core might be insufficient to break bonds in the rest of the cluster ion. Diatomics-in-molecules calculations by Doltsinis and Knowles ${ }^{35}$ yielded the threshold energies for the process $\mathrm{Ar}_{n}^{+} \rightarrow \mathrm{Ar}_{2}^{+}+(n-2)$ Ar to be $200 \mathrm{meV}$ for $n=3,280 \mathrm{meV}$ for $n=4,370 \mathrm{meV}$ for $n=5$, etc., and suggest that the fragmentation, if it happens, is unlikely to produce $\mathrm{Ar}_{2}^{+}$in the metastable II $(1 / 2)_{u}$ state.

To calculate the relative population of the rovibronic levels of the II $(1 / 2)_{u}$ electronic state, we assume vertical ionization of the neutral $\mathrm{Ar}_{2}$, i.e., the probability of the transition is proportional to Franck-Condon factors. ${ }^{36}$ Furthermore, we assume no change in the rotational quantum number during the ionization ${ }^{13}$ and a thermal population of the rovibrational levels in the neutral argon dimer. ${ }^{37}$ This allows us to calculate the relative rovibrational population in $\mathrm{Ar}_{2}^{+} \operatorname{II}(1 / 2)_{u}$ and thus to obtain the overall KERD for the metastable decay of $\mathrm{Ar}_{2}^{+}$.

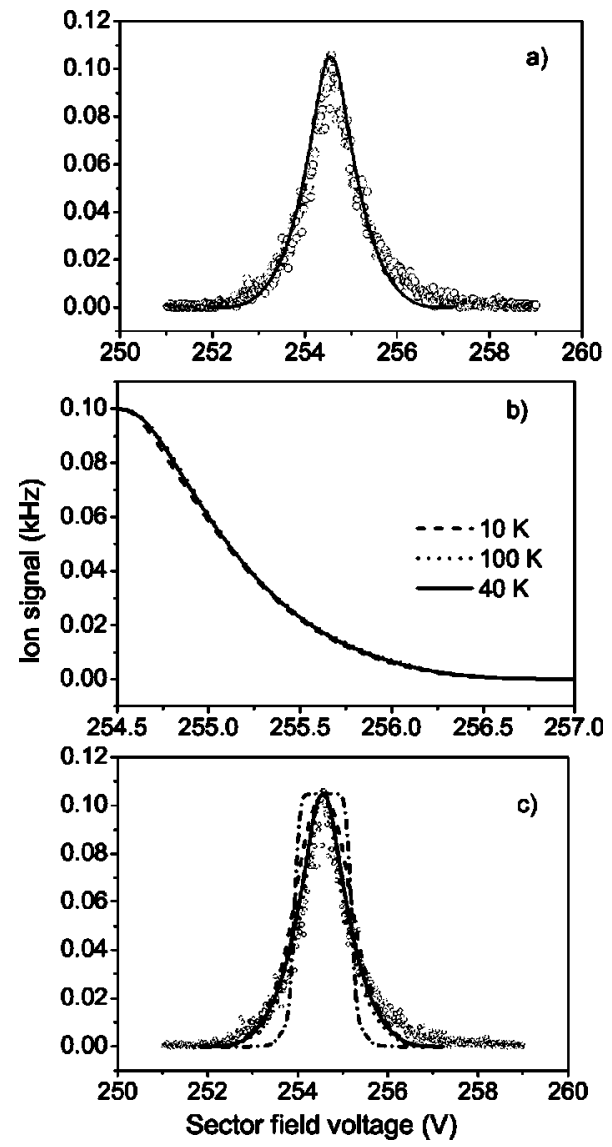

FIG. 4. (a) Experimental MIKE peak (open circles) in comparison with the one calculated using the potential energy curves from Fig. 3 (solid line). (b) Right halves of the MIKE peaks calculated for different vibrational temperatures of neutral $\mathrm{Ar}_{2}$. (c) MIKE peak calculated using different sets of $\mathrm{Ar}_{2}^{+}$ potential energy curves. Solid line-Wüest and Merkt (Ref. 24), dotted line-ab initio curves of Ha et al. (Ref. 23), dashed line-ab initio curves of Gadea and Paidarova (Ref. 22), dash-dotted line-Whitaker et al. (Ref. 13).

\section{Experiment vs calculation}

Figure 4(a) displays the MIKE peak computed from the potential energy curves of Wüest and Merkt ${ }^{24}$ as described above, together with the experimental MIKE peak. For the vibrational and rotational temperature of argon dimers in the supersonic beam we assumed $40 \mathrm{~K}$. This value derived from the Raman study of $\mathrm{Ar}_{2}$ by Godfried and Silvera ${ }^{37}$ is consistent with the measurement of the temperature of large argon clusters by electron diffraction. ${ }^{38}$ The excellent agreement between the experimental and computed MIKE peaks strongly suggests that the $\mathrm{II}(1 / 2)_{u} \rightarrow \mathrm{I}(1 / 2)_{g}$ transition indeed is the observed decay process.

Our calculations also explain why Yoshii et al. ${ }^{17} \mathrm{did}$ not observe any metastable decay in their experiment on $\mathrm{Ar}_{2}^{+}$. They photoionized $\mathrm{Ar}_{2}$ to produce $\mathrm{Ar}_{2}^{+}$in the $v=0$ vibrational level of the II $(1 / 2)_{u}$ electronic state. The rate of the continuum transitions into $\mathrm{I}(1 / 2)_{g}$ state for this level is very small, for example, it is about 80 times smaller than the transition rate from the $v=4$ level. Accordingly, the intensity for this transition is very low [Eq. (9)] in the time window sampled.

To show the effect of the $\mathrm{II}(1 / 2)_{u}$ rovibrational popula- 
tion on the calculated MIKE peak, the calculations were performed at different temperatures. Figure 4(b) shows the result. The influence of temperature is almost negligible-it turns out that the rovibronic population of the $\mathrm{II}(1 / 2)_{u}$ is of smaller importance than the relative magnitude of the transition rates corresponding to different rovibrational levels.

Another question is how much the potential energy curves used for the calculation of the transition rates influence the theoretical MIKE peak. In Fig. 4(c) we show four theoretical MIKE peaks, calculated from different sets of potential curves. The agreement with experiment is excellent for the potential energy curves of Wüest and Merkt, ${ }^{24}$ as well as for the $a b$ initio curves of Ha et $_{a l} .^{23}$ The results obtained using the potential energy curves of Gadea and Paidarova ${ }^{22}$ do not agree so well in the region of low kinetic energies (seen near the maximum of the MIKE peak). The MIKE peak calculated using the potential energy curves of Whitaker and co-workers ${ }^{13}$ is too wide; this is caused by an overestimation of the local maximum in the $\mathrm{I}(1 / 2)_{g}$ electronic state. Thus our approach represents a method for testing the accuracy of potential energy curves.

\section{SUMMARY AND CONCLUSIONS}

A comprehensive and systematic investigation of the metastable decay of $\mathrm{Ar}_{2}^{+}$has been presented in this work. Use was made of the experimental MIKE spectra for the spontaneous dissociation $\mathrm{Ar}_{2}^{+} \rightarrow \mathrm{Ar}^{+}+\mathrm{Ar}$ in the time regime of microseconds. Quantitative analysis of the possible origins for such a metastable dissociation shows that the only operative process with respect to lifetimes is the $\mathrm{II}(1 / 2)_{u}$ $\rightarrow \mathrm{I}(1 / 2)_{g}$ bound to continuum radiative transition. As an additional proof of this assignment, kinetic energy release distributions of fragment ions have been calculated and the theoretical shape of the fragment ion MIKE peak has been constructed from them. The present calculations show that the $\mathrm{II}(1 / 2)_{u} \rightarrow \mathrm{I}(1 / 2)_{g}$ bound to continuum radiative transition is indeed responsible for the occurrence of the observed decay process. Excellent agreement was obtained between the experimental MIKE peak and the theoretical predictions if the latter are based on the $\mathrm{Ar}_{2}^{+}$potential energy curves constructed by Wüest and Merkt. ${ }^{24}$

\section{ACKNOWLEDGMENTS}

The authors thank I. Paidarova and F. Merkt for providing potential curves of $\mathrm{Ar}_{2}^{+}$and F. Hagelberg and M. Probst for fruitful discussions. This work was partly supported by the FWF, ÖAW, and ÖNB, Wien, Austria, and the European Commission, Brussels.
${ }^{1}$ R. G. Cooks, J. H. Beynon, R. M. Caprioli, and R. G. Lester, Metastable Ions (Elsevier, Amsterdam, 1973).

${ }^{2}$ G. Herzberg, Molecular Spectra and Molecular Structure I. Spectra of Diatomic Molecules (Van Nostradam Reinhold, New York, 1950).

${ }^{3} \mathrm{~K}$. Levsen, Fundamental Aspects of Organic Mass Spectrometry (Chemie, Weinheim, 1975).

${ }^{4}$ J. G. Eden, IEEE J. Sel. Top. Quantum Electron. 6, 1051 (2000).

${ }^{5}$ J. J. Ewing, IEEE J. Sel. Top. Quantum Electron. 6, 1061 (2000).

${ }^{6}$ J. A. R. Samson, Techniques of Vacuum Ultraviolet Spectroscopy (Pied Publications, Lincoln, NE, 1980).

${ }^{7}$ J. Galindez, F. Calvo, P. Paska, D. Hrivnak, R. Kalus, and F. X. Gadea, Comput. Phys. Commun. 154, 126 (2002).

${ }^{8}$ F. Calvo, J. Galindez, and F. X. Gadea, Phys. Chem. Chem. Phys., 5, 321 (2003).

${ }^{9}$ F. Y. Naumkin, Chem. Phys. 252, 301 (2000).

${ }^{10}$ N. L. Doltsinis and P. J. Knowles, Chem. Phys. Lett. 325, 648 (2000).

${ }^{11}$ K. Stephan, A. Stamatovic, and T. D. Märk, Phys. Rev. A 28, 3105 (1983).

${ }^{12}$ W. R. Wadt, J. Chem. Phys. 68, 402 (1978).

${ }^{13}$ B. J. Whitaker, C. A. Woodward, P. J. Knowles, and A. J. Stace, J. Chem. Phys. 93, 376 (1990).

${ }^{14}$ K. Stephan and T. D. Märk, Phys. Rev. A 32, 1447 (1985).

${ }^{15}$ K. Norwood, J. H. Guo, and C. Y. Ng, J. Chem. Phys. 90, 2995 (1989).

${ }^{16}$ A. J. Stace, C. A. Woodward, and B. J. Whitaker, Chem. Phys. Lett. 184, 113 (1991).

${ }^{17}$ H. Yoshii, T. Hayaishi, T. Onuma, T. Aoto, Y. Morioka, and K. Ito, J. Chem. Phys. 117, 1517 (2002).

${ }^{18}$ J. Fedor, R. Parajuli, S. Matt-Leubner et al., Phys. Rev. Lett. 91, 133401 (2003).

${ }^{19}$ S. Matt, M. Sonderegger, R. David, O. Echt, P. Scheier, J. Laskin, C. Lifshitz, and T. D. Märk, Int. J. Mass. Spectrom. 187, 813 (1999).

${ }^{20}$ S. Matt-Leubner, A. Stamatovic, R. Parajuli, P. Scheier, T. D. Märk, O. Echt, and C. Lifshitz, Int. J. Mass. Spectrom. 222, 213 (2003).

${ }^{21}$ H. H. Michels, R. H. Hobbs, and L. A. Wright, J. Chem. Phys. 69, 5151 (1978).

${ }^{22}$ F. X. Gadea and I. Paidarova, Chem. Phys. 209, 281 (1996).

${ }^{23}$ T. K. Ha, R. Rupper, A. Wüest, and F. Merkt, Mol. Phys. 101, 827 (2003).

${ }^{24}$ A. Wüest and F. Merkt, J. Chem. Phys. 120, 638 (2004).

${ }^{25}$ J. C. Houver, J. Baudon, M. Abignoli, M. Barat, P. Fournier, and J. Durup, Int. J. Mass Spectrom. Ion Phys. 4, 137 (1970).

${ }^{26}$ R. J. Le Roy and W. K. Liu, J. Chem. Phys. 69, 3622 (1978).

${ }^{27}$ R. J. Le Roy and R. B. Bernstein, J. Chem. Phys. 54, 5114 (1971).

${ }^{28}$ J. S. Townsend, A Modern Approach to Quantum Mechanics (University Science, Sausalito, CA, 2000).

${ }^{29}$ A. Carrington and T. P. Softley, Chem. Phys. 92, 199 (1985).

${ }^{30}$ R. Loudon, The Quantum Theory of Light (Clarendon, Oxford, 1992).

${ }^{31}$ R. A. Buckingham, in Quantum Theory I, Elements, edited by D. R. Bates (Academic, New York, 1961).

${ }^{32}$ U. Buck and H. Meyer, J. Chem. Phys. 84, 4854 (1986).

${ }^{33}$ O. F. Hagena and W. Obert, J. Chem. Phys. 56, 1793 (1972).

${ }^{34} \mathrm{H}$. Haberland, Clusters of Atoms and Molecules (Springer, Berlin, 1994).

${ }^{35}$ N. L. Doltsinis and P. J. Knowles, Mol. Phys. 94, 981 (1998).

${ }^{36} \mathrm{~S}$. M. Younger and T. D. Märk, in Electron Impact Ionization, edited by T. D. Märk, G. H. Dunn (Springer New York, 1985).

${ }^{37}$ H. P. Godfried and I. F. Silvera, Phys. Rev. A 27, 3008 (1982).

${ }^{38}$ J. Farges, M. F. Deferaudy, B. Raoult, and B. Torchet, Surf. Sci. 106, 95 (1981). 